\title{
The energy dependence of $p_{t}$ angular correlations inferred from mean- $p_{t}$ fluctuation scale dependence in heavy ion collisions at the SPS and RHIC
}

\author{
STAR Collaboration \\ J Adams ${ }^{1}$, M M Aggarwal ${ }^{2}$, Z Ahammed ${ }^{3}$, J Amonett ${ }^{4}$, B D Anderson ${ }^{4}$, \\ M Anderson ${ }^{5}$, D Arkhipkin ${ }^{6}$, G S Averichev ${ }^{7}$, Y Bai $^{8}$, J Balewski ${ }^{9}$, \\ O Barannikova ${ }^{10}$, L S Barnby ${ }^{1}$, J Baudot ${ }^{11}$, S Bekele ${ }^{12}$, V V Belaga ${ }^{7}$, \\ A Bellingeri-Laurikainen ${ }^{13}$, R Bellwied ${ }^{14}$, B I Bezverkhny ${ }^{15}$, \\ S Bhardwaj ${ }^{16}$, A Bhasin ${ }^{17}$, A K Bhati ${ }^{2}$, H Bichsel ${ }^{18}$, J Bielcik ${ }^{15}$, \\ J Bielcikova ${ }^{15}$, L C Bland ${ }^{19}$, C O Blyth ${ }^{1}$, S-L Blyth ${ }^{20}$, B E Bonner ${ }^{21}$, \\ M Botje ${ }^{8}$, J Bouchet ${ }^{13}$, A V Brandin ${ }^{22}$, A Bravar ${ }^{19}$, M Bystersky ${ }^{23}$, \\ R V Cadman ${ }^{24}$, X Z Cai ${ }^{25}$, H Caines ${ }^{15}$, M Calderón de la Barca Sánchez ${ }^{5}$, \\ J Castillo $^{8}$, O Catu ${ }^{15}$, D Cebra ${ }^{5}$, Z Chajecki ${ }^{12}$, P Chaloupka ${ }^{23}$, \\ S Chattopadhyay ${ }^{3}$, H F Chen ${ }^{26}$, J H Chen ${ }^{25}$, Y Chen ${ }^{27}$, J Cheng ${ }^{28}$, \\ M Cherney $^{29}$, A Chikanian ${ }^{15}$, H A Choi ${ }^{30}$, W Christie ${ }^{19}$, J P Coffin ${ }^{11}$,

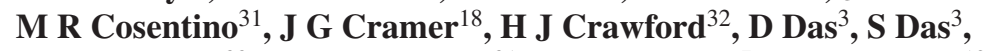 \\ M Daugherity ${ }^{33}$, M M de Moura ${ }^{31}$, T G Dedovich ${ }^{7}$, M DePhillips ${ }^{19}$, \\ A A Derevschikov ${ }^{34}, \mathbf{L}_{\text {Didenko }}{ }^{19}$, T Dietel $^{35}$, P Djawotho $^{9}, \mathbf{S}$ M Dogra ${ }^{17}$, \\ W J Dong ${ }^{27}$, X Dong ${ }^{26}$, J E Draper ${ }^{5}$, F Du ${ }^{15}$, V B Dunin ${ }^{7}$, J C Dunlop ${ }^{19}$, \\ M R Dutta Mazumdar ${ }^{3}, \mathrm{~V}$ Eckardt $^{36}$, W R Edwards ${ }^{20}$, L G Efimov Ed, $^{7}$ \\ V Emelianov ${ }^{22}$, J Engelage ${ }^{32}$, G Eppley ${ }^{21}$, B Erazmus ${ }^{13}$, M Estienne ${ }^{11}$, \\ P Fachini $^{19}$, R Fatemi ${ }^{37}$, J Fedorisin ${ }^{7}$, K Filimonov ${ }^{20}$, P Filip $^{6}$, E Finch ${ }^{15}$, \\ V Fine ${ }^{19}$, Y Fisyak ${ }^{19}$, J Fu ${ }^{38}, \mathbf{C}$ A Gagliardi ${ }^{39}$, L Gaillard ${ }^{1}$, J Gans $^{15}$, \\ M S Ganti ${ }^{3}$, V Ghazikhanian ${ }^{27}$, P Ghosh ${ }^{3}$, J E Gonzalez ${ }^{27}$, \\ Y G Gorbunov ${ }^{29}$, H Gos $^{40}$, O Grebenyuk ${ }^{8}$, D Grosnick ${ }^{41}$, S M Guertin ${ }^{27}$, \\ K S F F Guimaraes ${ }^{31}$, Y Guo ${ }^{14}$, N Gupta ${ }^{17}$, T D Gutierrez ${ }^{5}$, B Haag Ha $^{5}$ \\ T J Hallman ${ }^{19}$, A Hamed ${ }^{14}$, J W Harris ${ }^{15}$, W He H $^{9}$, Meinz ${ }^{15}$, \\ T W Henry ${ }^{39}$, S Hepplemann ${ }^{42}$, B Hippolyte ${ }^{11}$, A Hirsch ${ }^{10}$, E Hjort ${ }^{20}$, \\ G W Hoffmann ${ }^{33}$, M J Horner ${ }^{20}$, H Z Huang ${ }^{27}$, S L Huang ${ }^{26}$, \\ E W Hughes ${ }^{43}$, T J Humanic ${ }^{12}$, G Igo ${ }^{27}$, P Jacobs ${ }^{20}$, W W Jacobs ${ }^{9}$, \\ P Jakl ${ }^{23}$, F Jia ${ }^{44}$, H Jiang ${ }^{27}$, P G Jones ${ }^{1}$, E G Judd ${ }^{32}$, S Kabana ${ }^{13}$, \\ K Kang ${ }^{28}$, J Kapitan ${ }^{23}$, M Kaplan ${ }^{45}$, D Keane ${ }^{4}$, A Kechechyan ${ }^{7}$, \\ V Yu Khodyrev ${ }^{34}$, B C Kim ${ }^{30}$, J Kiryluk ${ }^{37}$, A Kisiel ${ }^{40}$, E M Kislov ${ }^{7}$, \\ D D Koetke ${ }^{41}$, T Kollegger ${ }^{35}$, M Kopytine ${ }^{4}$, L Kotchenda ${ }^{22}$, V Kouchpil ${ }^{23}$, \\ K L Kowalik ${ }^{20}$, M Kramer ${ }^{46}$, P Kravtsov ${ }^{22}$, V I Kravtsov ${ }^{34}$, K Krueger ${ }^{24}$, \\ C Kuhn ${ }^{11}$, A I Kulikov ${ }^{7}$, A Kumar ${ }^{2}$, A A Kuznetsov ${ }^{7}$, M A C Lamont ${ }^{15}$, \\ J M Landgraf ${ }^{19}$, S Lange ${ }^{35}$, S LaPointe ${ }^{14}$, F Laue ${ }^{19}$, J Lauret ${ }^{19}$, \\ A Lebedev ${ }^{19}, \mathbf{R}$ Lednicky $^{6}, \mathrm{C}-\mathrm{H}$ Lee $^{30}, \mathbf{S}$ Lehocka $^{7}, \mathbf{M} \mathbf{J}$ LeVine ${ }^{19}, \mathbf{C ~ L i}^{26}$, \\ $\mathbf{Q ~ L i}{ }^{14}, \mathbf{Y ~ L i}{ }^{28}, \mathbf{G ~ L i n}{ }^{15}, \mathbf{S ~ J ~ L i n d e n b a u m}{ }^{46}, \mathbf{M ~ A ~ L i s a}{ }^{12}, \mathbf{F ~ L i u}{ }^{38}, \mathbf{H ~ L i u}^{26}$, \\ J Liu ${ }^{21}$, L Liu ${ }^{38}$, Z Liu ${ }^{38}$, T Ljubicic ${ }^{19}$, W J Llope ${ }^{21}$, H Long ${ }^{27}$, \\ R S Longacre ${ }^{19}$, M Lopez-Noriega ${ }^{12}$, W A Love ${ }^{19}$, Y Lu ${ }^{38}$, T Ludlam ${ }^{19}$,
}


D Lynn ${ }^{19}$, G L Ma ${ }^{25}$, J G Ma ${ }^{27}$, Y G Ma ${ }^{25}$, D Magestro ${ }^{12}$, D P Mahapatra ${ }^{47}$, R Majka ${ }^{15}$, L K Mangotra ${ }^{17}$, R Manweiler $^{41}$, S Margetis ${ }^{4}$, C Markert ${ }^{4}$, L Martin ${ }^{13}$, H S Matis ${ }^{20}$, Yu A Matulenko ${ }^{34}$, C J McClain 24, T S McShane ${ }^{29}$, Yu Melnick ${ }^{34}$, A Meschanin ${ }^{34}$, M L Miller ${ }^{37}$, N G Minaev ${ }^{34}$, S Mioduszewski ${ }^{39}$, C Mironov $^{4}$, A Mischke ${ }^{8}$, D K Mishra ${ }^{47}$, J Mitchell ${ }^{21}$, B Mohanty ${ }^{3}$, L Molnar ${ }^{10}$, C F Moore ${ }^{33}$, D A Morozov ${ }^{34}$, M G Munhoz ${ }^{31}$, B K Nandi ${ }^{48}$, C Nattrass $^{15}$, T K Nayak ${ }^{3}$, J M Nelson ${ }^{1}$, P K Netrakanti ${ }^{3}$, V A Nikitin ${ }^{6}$, L V Nogach ${ }^{34}$, S B Nurushev ${ }^{34}$, G Odyniec ${ }^{20}$, A Ogawa ${ }^{19}$, V Okorokov ${ }^{22}$, M Oldenburg ${ }^{20}$,

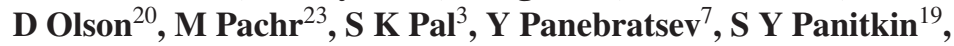
A I Pavlinov ${ }^{14}$, T Pawlak ${ }^{40}$, T Peitzmann ${ }^{8}$, V Perevoztchikov ${ }^{19}$, C Perkins $^{32}$, W Peryt ${ }^{40}$, V A Petrov ${ }^{14}$, S C Phatak ${ }^{47}$, R Picha ${ }^{5}$, M Planinic ${ }^{49}$, J Pluta ${ }^{40}$, N Poljak ${ }^{49}$, N Porile ${ }^{10}$, J Porter $^{18}$, M Potekhin $^{19}$, E Potrebenikova ${ }^{7}$, B V K S Potukuchi ${ }^{17}$, D Prindle ${ }^{18}$, J Putschke ${ }^{20}$, G Rakness ${ }^{42}$, R Raniwala ${ }^{16}$, S Raniwala ${ }^{16}$, R L Ray ${ }^{33}$, S V Razin ${ }^{7}$, J Reinnarth ${ }^{13}$, D Relyea ${ }^{43}$, F Retiere ${ }^{20}$, A Ridiger ${ }^{22}$, H G Ritter ${ }^{20}$, J B Roberts ${ }^{21}$, O V Rogachevskiy ${ }^{7}$, J L Romero ${ }^{5}$, A Rose ${ }^{20}$, C Roy $^{13}$, L Ruan ${ }^{20}$, M J Russcher ${ }^{8}$, R Sahoo ${ }^{47}$, I Sakrejda ${ }^{20}$, S Salur ${ }^{15}$, J Sandweiss $^{15}$, M Sarsour ${ }^{39}$, P S Sazhin ${ }^{7}$, J Schambach ${ }^{33}$, R P Scharenberg ${ }^{10}$, N Schmitz $^{36}$, K Schweda $^{20}$, J Seger $^{29}$, I Selyuzhenkov ${ }^{14}$, P Seyboth $^{36}$, A Shabetai ${ }^{20}$, E Shahaliev ${ }^{7}$, M Shao $^{26}$, M Sharma $^{2}$, W Q Shen ${ }^{25}$, S S Shimanskiy ${ }^{7}$, E Sichtermann ${ }^{20}$, F Simon ${ }^{37}$, R N Singaraju ${ }^{3}, \mathbf{N}$ Smirnov ${ }^{15}$, R Snellings $^{8}$, G Sood $^{41}$, P Sorensen $^{19}$, J Sowinski ${ }^{9}$, J Speltz ${ }^{11}$, H M Spinka ${ }^{24}$, B Srivastava ${ }^{10}$, A Stadnik ${ }^{7}$, T D S Stanislaus ${ }^{41}$, R Stock ${ }^{35}$, A Stolpovsky ${ }^{14}$, M Strikhanov ${ }^{22}$, B Stringfellow ${ }^{10}$, A A P Suaide ${ }^{31}$, E Sugarbaker ${ }^{12}$, M Sumbera ${ }^{23}$, Z Sun $^{44}$, B Surrow $^{37}$, M Swanger ${ }^{29}$, T J M Symons ${ }^{20}$, A Szanto de Toledo ${ }^{31}$, A Tai $^{27}$, J Takahashi ${ }^{31}$, A H Tang ${ }^{19}$, T Tarnowsky ${ }^{10}$, D Thein ${ }^{27}$, J H Thomas ${ }^{20}$, A R Timmins ${ }^{1}$, S Timoshenko ${ }^{22}$, M Tokarev ${ }^{7}$, T A Trainor ${ }^{18}$, S Trentalange ${ }^{27}$, R E Tribble ${ }^{39}$, O D Tsai ${ }^{27}$, J Ulery ${ }^{10}$, T Ullrich ${ }^{19}$, D G Underwood ${ }^{24}$, G Van Buren ${ }^{19}$, N van der Kolk ${ }^{8}$, M van Leeuwen ${ }^{20}, \mathbf{R}$ Varma $^{48}$, I M Vasilevski ${ }^{6}, \mathbf{A}$ N Vasiliev ${ }^{34}, \mathbf{R}_{\text {Vernet }^{11}}$, S E Vigdor ${ }^{9}$, Y P Viyogi ${ }^{3}$, S Vokal $^{7}$, W T Waggoner ${ }^{29}$, F Wang ${ }^{10}$, G Wang ${ }^{4}$, J S Wang ${ }^{44}$, X L Wang ${ }^{26}$, Y Wang ${ }^{28}$, J W Watson ${ }^{4}$, J C Webb ${ }^{9}$, G D Westfall ${ }^{50}$, A Wetzler ${ }^{20}$, C Whitten $\mathbf{J}^{27}$, H Wieman ${ }^{20}, \mathbf{S}$ W Wissink ${ }^{9}$, R Witt ${ }^{15}, \mathbf{J}$ Wood $^{27}, \mathbf{J} \mathbf{W u}^{26}, \mathbf{N} \mathbf{X u}^{20}, \mathbf{Q} \mathbf{H} \mathbf{X u}^{20}, \mathbf{Z}$ Xu ${ }^{19}$, P Yepes $^{21}$, I-K Yoo ${ }^{30}$, V I Yurevich ${ }^{7}$, W Zhan ${ }^{44}$, H Zhang ${ }^{19}$, W M Zhang ${ }^{4}$, Y Zhang ${ }^{26}$, Z P Zhang ${ }^{26}$, Y Zhao ${ }^{26}$, C Zhong ${ }^{25}$, R Zoulkarneev ${ }^{6}$, Y Zoulkarneeva ${ }^{6}$, A N Zubarev ${ }^{7}$ and J X Zuo $\mathbf{Z u}^{25}$

\footnotetext{
${ }^{1}$ University of Birmingham, Birmingham, UK

${ }^{2}$ Panjab University, Chandigarh 160014 , India

${ }^{3}$ Variable Energy Cyclotron Centre, Kolkata 700 064, India

${ }^{4}$ Kent State University, Kent, OH 44242, USA

${ }^{5}$ University of California, Davis, CA 95616, USA

${ }^{6}$ Particle Physics Laboratory (JINR), Dubna, Russia

${ }^{7}$ Laboratory for High Energy (JINR), Dubna, Russia

${ }^{8}$ NIKHEF and Utrecht University, Amsterdam, The Netherlands

${ }^{9}$ Indiana University, Bloomington, IN 47408, USA

${ }^{10}$ Purdue University, West Lafayette, IN 47907, USA

${ }^{11}$ Institut de Recherches Subatomiques, Strasbourg, France
} 
${ }^{12}$ Ohio State University, Columbus, OH 43210, USA

${ }^{13}$ SUBATECH, Nantes, France

14 Wayne State University, Detroit, MI 48201, USA

15 Yale University, New Haven, CT 06520, USA

${ }^{16}$ University of Rajasthan, Jaipur 302004 , India

${ }^{17}$ University of Jammu, Jammu 180001 , India

18 University of Washington, Seattle, WA 98195, USA

${ }^{19}$ Brookhaven National Laboratory, Upton, NY 11973, USA

${ }^{20}$ Lawrence Berkeley National Laboratory, Berkeley, CA 94720, USA

${ }^{21}$ Rice University, Houston, TX 77251, USA

22 Moscow Engineering Physics Institute, Moscow, Russia

${ }^{23}$ Nuclear Physics Institute AS CR, 25068 Řež/Prague, Czech Republic

${ }^{24}$ Argonne National Laboratory, Argonne, IL 60439, USA

25 Shanghai Institute of Applied Physics, Shanghai 201800, People's Republic of China

${ }^{26}$ University of Science and Technology of China, Hefei 230026, People's Republic of China

27 University of California, Los Angeles, CA 90095, USA

28 Tsinghua University, Beijing 100084, People's Republic of China

${ }^{29}$ Creighton University, Omaha, NE 68178, USA

${ }^{30}$ Pusan National University, Pusan, Korea

${ }^{31}$ Universidade de Sao Paulo, Sao Paulo, Brazil

32 University of California, Berkeley, CA 94720, USA

${ }^{33}$ University of Texas, Austin, TX 78712, USA

34 Institute of High Energy Physics, Protvino, Russia

${ }^{35}$ University of Frankfurt, Frankfurt, Germany

${ }^{36}$ Max-Planck-Institut für Physik, Munich, Germany

${ }^{37}$ Massachusetts Institute of Technology, Cambridge, MA 02139-4307, USA

${ }^{38}$ Institute of Particle Physics, CCNU (HZNU), Wuhan 430079, People's Republic of China

39 Texas A\&M University, College Station, TX 77843, USA

${ }^{40}$ Warsaw University of Technology, Warsaw, Poland

41 Valparaiso University, Valparaiso, IN 46383, USA

42 Pennsylvania State University, University Park, PA 16802, USA

${ }^{43}$ California Institute of Technology, Pasadena, CA 91125, USA

44 Institute of Modern Physics, Lanzhou, People's Republic of China

${ }^{45}$ Carnegie Mellon University, Pittsburgh, PA 15213, USA

${ }^{46}$ City College of New York, New York City, NY 10031, USA

${ }^{47}$ Institute of Physics, Bhubaneswar 751005 , India

48 Indian Institute of Technology, Mumbai, India

${ }^{49}$ University of Zagreb, Zagreb, HR-10002, Croatia

${ }^{50}$ Michigan State University, East Lansing, MI 48824, USA

Received 20 September 2006

Published 18 January 2007

Online at stacks.iop.org/JPhysG/34/451

\section{Abstract}

We present the first study of the energy dependence of $p_{t}$ angular correlations inferred from event-wise mean transverse momentum $\left\langle p_{t}\right\rangle$ fluctuations in heavy ion collisions. We compare our large-acceptance measurements at CM energies $\sqrt{s_{\mathrm{NN}}}=19.6,62.4,130$ and $200 \mathrm{GeV}$ to SPS measurements at 12.3 and 17.3 GeV. $p_{t}$ angular correlation structure suggests that the principal source of $p_{t}$ correlations and fluctuations is minijets (minimum-bias parton fragments). We observe a dramatic increase in correlations and fluctuations from SPS to RHIC energies, increasing linearly with $\ln \sqrt{s_{\mathrm{NN}}}$ from the onset of observable jet-related $\left\langle p_{t}\right\rangle$ fluctuations near $10 \mathrm{GeV}$.

(Some figures in this article are in colour only in the electronic version) 


\section{Introduction}

Theoretical descriptions of heavy ion (HI) collisions at RHIC energies predict copious parton (mainly gluon) production in the early stages of collisions and subsequent parton rescattering as the principal route to a colour-deconfined bulk medium, with possible equilibration to a quark-gluon plasma [1-5]. Particle yields, spectra and high- $p_{t}$ correlations from $\mathrm{Au}-\mathrm{Au}$ collisions at 130 and $200 \mathrm{GeV}$ provide tantalizing evidence that a QCD coloured medium is indeed produced at RHIC [6-11]. What are the properties of that medium, and has an equilibrated QGP formed prior to hadronic decoupling? A partial answer may emerge by searching for evidence of initial-state semi-hard scattered partons in the correlations and fluctuations of final-state hadrons. In particular, $p_{t}$ fluctuations and correlations may provide such evidence.

Fluctuations (variations about a mean) of event-wise mean transverse momentum $\left\langle p_{t}\right\rangle$ $[12,13]$ within momentum-space angular bins of varying size, and corresponding two-particle $p_{t}$ correlations (variation of a two-particle distribution relative to a reference), could provide access to early parton scattering and subsequent in-medium dissipation inaccessible by other means $[14]^{51}$. $p_{t}$ correlations may represent such partons in the form of local velocity and/or temperature correlations $[15,16]$. Measurements of $\left\langle p_{t}\right\rangle$ fluctuations in $\mathrm{Au}-\mathrm{Au}$ collisions at fixed scale (bin size) at $130 \mathrm{GeV}$ [12] and measurements of $p_{t}$ angular correlations inferred from $\left\langle p_{t}\right\rangle$ fluctuation scale dependence at $200 \mathrm{GeV}$ [17] indicate that $p_{t}$ correlations at RHIC are much larger than those at the SPS.

In this paper we report the first study of the energy dependence of $p_{t}$ angular correlations (e.g., structures in the event-wise $p_{t}$ distribution on $(\eta, \phi)$ which occur at different positions in each HI collision) inferred from excess $\left\langle p_{t}\right\rangle$ fluctuations (fluctuations beyond those expected for independent particle $p_{t}$ production). We present the scale dependence of $\left\langle p_{t}\right\rangle$ fluctuations within the STAR detector acceptance for four RHIC energies and provide a basis for interpreting those fluctuations by inverting the fluctuation scale dependence for two energies and two centralities to form $p_{t}$ autocorrelations on angle space $(\eta, \phi)$. We obtain the centrality dependence of full-acceptance fluctuations at four RHIC energies compared to results at two SPS energies, and we determine the $\sqrt{s_{\mathrm{NN}}}$ dependence of $\left\langle p_{t}\right\rangle$ fluctuations for full-acceptance STAR data as a basis for comparison with extrapolated CERES measurements [18] and the pQCD event simulation Monte Carlo Hijing [19]. This analysis is based on $\mathrm{Au}-\mathrm{Au}$ collisions observed with the STAR detector at the Relativistic Heavy Ion Collider (RHIC).

\section{Analysis method}

Excess charge-independent (all charged particles combined) $\left\langle p_{t}\right\rangle$ fluctuations are measured by the difference between the variance of quantity $\left\{p_{t}(\delta x)-n(\delta x) \hat{p}_{t}\right\} / \sqrt{\bar{n}(\delta x)}$ and the variance $\sigma_{\hat{p}_{t}}^{2}$ of an uncorrelated reference [12]. $p_{t}(\delta x)$ is the scalar sum of $p_{t}$ in a bin of size $\delta x$ (e.g., $\delta \eta$ or $\delta \phi), n(\delta x)$ is the number of particles in the bin, and $\hat{p}_{t}$ is the mean and $\sigma_{\hat{p}_{t}}^{2}$ is the variance of the single-particle $p_{t}$ spectrum for all accepted charged particles from all events $\left(\hat{p}_{t}\right.$ and $\sigma_{\hat{p}_{t}}^{2}$ then represent independent particle $p_{t}$ production). The variance difference

$$
\begin{aligned}
\Delta \sigma_{p_{t}: n}^{2}(\delta x) & \equiv \overline{\left\{p_{t}(\delta x)-n(\delta x) \hat{p}_{t}\right\}^{2}} / \bar{n}(\delta x)-\sigma_{\hat{p}_{t}}^{2} \\
& \equiv 2 \sigma_{\hat{p}_{t}} \Delta \sigma_{p_{t}: n}(\delta x),
\end{aligned}
$$

${ }^{51}$ It is argued that elliptic flow as described by hydro models is sensitive to initial parton scattering and subsequent equilibration. However, the supporting arguments contain elements not directly confirmed by experiment. Experimental observation of minijet correlations and their evolution with A-A centrality may provide more direct access to the early dynamics of heavy ion collisions. 
calculated over a range of bin sizes is the scale-dependent $\left\langle p_{t}\right\rangle$ fluctuation measure for this analysis [17]. Overlines indicate averages over all bins with size $\delta x$ in all events. The difference factor $\Delta \sigma_{p_{t}: n}$ is related to the $\left\langle p_{t}\right\rangle$ fluctuation measure $\Phi_{p_{t}}$ introduced previously [20-22] by $\Delta \sigma_{p_{t}: n}=\Phi_{p_{t}}\left(1+\Phi_{p_{t}} / 2 \sigma_{\hat{p}_{t}}\right)$.

Event-wise fluctuations in the bin contents of a binned distribution reflect changes in the structure of that distribution. Smaller bins are sensitive to more local aspects of that structure, and conversely. The scale dependence of fluctuations is therefore equivalent in some sense to a running integral of the corresponding two-particle distribution, the integration limit determined by the bin size or scale [23, 24]. The integrand is an autocorrelation which compares a distribution $f(x)$ to itself. An autocorrelation is effectively a projection by averaging of product distribution $f\left(x_{1}\right) \cdot f\left(x_{2}\right)$ on $\left(x_{1}, x_{2}\right)$ onto the difference variable $x_{\Delta} \equiv x_{1}-x_{2} \cdot{ }^{52}$ In this analysis we wish to determine the average angular correlation structure of the event-wise $p_{t}$ distribution on $(\eta, \phi)$ : what are the aspects of that distribution which vary event-wise but which nevertheless have persistence and universality. We do so by inverting the scale dependence of excess $\left\langle p_{t}\right\rangle$ fluctuations (the integral) to obtain the autocorrelation of the $p_{t}$ distribution on $(\eta, \phi)[17,23,24]$.

Equation (2) is an integral equation in a discrete form which relates variance difference $\Delta \sigma_{p_{t}: n}^{2}(\delta \eta, \delta \phi)$ on pseudorapidity $\eta$ and azimuth angle $\phi$ to an autocorrelation distribution on $\left(\eta_{\Delta}, \phi_{\Delta}\right)$ (e.g., $\left.\eta_{\Delta} \equiv \eta_{1}-\eta_{2}\right)[17,23]$. The autocorrelation (cf figure 2 for example) compactly represents two-particle correlations on $(\eta, \phi)$ in HI collisions [25]. The 2D discrete integral equation is

$$
\Delta \sigma_{p_{t}: n}^{2}\left(m \epsilon_{\eta}, n \epsilon_{\phi}\right)=4 \sum_{k, l=1}^{m, n} \epsilon_{\eta} \epsilon_{\phi} K_{m n ; k l} \frac{\Delta \rho\left(p_{t}: n ; k \epsilon_{\eta}, l \epsilon_{\phi}\right)}{\sqrt{\rho_{\mathrm{ref}}\left(n ; k \epsilon_{\eta}, l \epsilon_{\phi}\right)}},
$$

with kernel $K_{m n ; k l} \equiv(m-k+1 / 2) / m \cdot(n-l+1 / 2) / n$ and fixed microbin sizes $\epsilon_{\eta}$ and $\epsilon_{\phi}$ for the discrete integral. That equation can be inverted (solved for the integrand) to obtain autocorrelation density ratio $\Delta \rho\left(p_{t}: n\right) / \sqrt{\rho_{\text {ref }}(n)}$ (units $\left.(\mathrm{GeV} / c)^{2}\right)$ as a per-particle $p_{t}$ correlation measure on $\left(\eta_{\Delta}, \phi_{\Delta}\right)$ from $\left\langle p_{t}\right\rangle$ fluctuation scale dependence of $\Delta \sigma_{p_{t}: n}^{2}(\delta \eta, \delta \phi)$ $[17,23] . \Delta \rho\left(p_{t}: n\right)$ is proportional to the average of $\left(p_{t}-n \hat{p}_{t}\right)$ covariances for all pairs of bins $\left(\eta_{\Delta}, \phi_{\Delta}\right)$ apart. $\sqrt{\rho_{\text {ref }}(n)}$ is the geometric mean of particle densities in those bins [17]. Density ratio $\Delta \rho\left(p_{t}: n\right) / \sqrt{\rho_{\text {ref }}(n)}$ is thus proportional to normalized covariance $\overline{\left(p_{t}-n \hat{p}_{t}\right)_{a}\left(p_{t}-n \hat{p}_{t}\right)_{b}} / \sqrt{\bar{n}_{a} \bar{n}_{b}}$ (averaged over certain bin combinations $(a, b)$ to form an autocorrelation). The density ratio has the form of Pearson's correlation coefficient [26], but with number variances in the denominator replaced by Poisson values $\bar{n}_{a}, \bar{n}_{b}$. The density ratio is derived and discussed in [23, 27-29].

\section{Data}

Data for this analysis were obtained with the STAR detector [30] using a $0.5 \mathrm{~T}$ uniform magnetic field parallel to the beam axis. Event triggering and charged-particle measurements with the time projection chamber (TPC) are described in [30]. Track definitions, tracking efficiencies, quality cuts and primary-particle definition are described in $[12,31]$. Tracks were accepted with pseudorapidity in the range $|\eta|<1$, transverse momentum in the range $p_{t} \in[0.15,2] \mathrm{GeV} / c$ and $2 \pi$ azimuth, defining the detector acceptance for this analysis. Particle identification was not implemented. Centrality classes (percentages of the total

\footnotetext{
52 Difference variable $x_{\Delta}=x_{1}-x_{2}$ represents a diagonal coordinate axis of 2D space $\left(x_{1}, x_{2}\right)$, the other diagonal
} being sum variable $x_{\Sigma}=x_{1}+x_{2}$. Coordinate axis $x_{\Delta}$ is differentiated from interval $\Delta x=x_{1}-x_{2}$ on 1D space $x$. 
hadronic cross section) were defined in terms of the uncorrected number $N$ of charged particles in acceptance $|\eta|<1$ according to procedures described in [12,17].

Centrality specified in terms of pathlength $v$ (estimating the mean number of nucleons encountered by a participant nucleon) is based on the relationship of $N$ to minimum-bias distribution endpoints $N_{p}$ and $N_{0} . \quad N_{p}$, the lower half-maximum point of the minimumbias distribution plotted as $\mathrm{d} \sigma / \mathrm{d} N^{1 / 4}$, estimates (one-half) the uncorrected mean multiplicity for non-single-diffractive nucleon-nucleon collisions in the same acceptance. $N_{0}$, the upper half-maximum point of $\mathrm{d} \sigma / \mathrm{d} N^{1 / 4}$, estimates the value of $N$ corresponding to the maximum number of participant nucleons $N_{\text {part,max }}$ and impact parameter $b=0$. The relation between the fractional cross section $\sigma / \sigma_{0}$ and multiplicity $N$ is approximated by expression $1-\sigma / \sigma_{0}=\left(N^{1 / 4}-N_{p}^{1 / 4}\right) /\left(N_{0}^{1 / 4}-N_{p}^{1 / 4}\right)[32]$, accurate to $\sim 3 \%$ over the entire centrality range (exluding fluctuations near the endpoints). Mean participant path length $v \approx 2 N_{\text {bin }} /$ $N_{\text {part }}{ }^{53}$ is then given by $v=\left\{1+2.23\left(1-\sigma / \sigma_{0}\right)\right\}^{6} /\left\{\left(1+2.72\left(1-\sigma / \sigma_{0}\right)\right\}^{4}\right.$ to about $2 \%$, based on a Monte Carlo Glauber simulation [32]. Coefficients 2.23 and 2.72 apply to $200 \mathrm{GeV}$ collisions and vary slowly with $\ln \left(\sqrt{s_{\mathrm{NN}}}\right)$, resulting in a few-percent shift in $v_{\max }$ with collision energy over the energy range $62-200 \mathrm{GeV}$. Those expressions determined the values of $v$ used in figure 3 (right panel).

\section{Fluctuations and correlations}

Figure 1 compares the scale dependence of variance difference $\Delta \sigma_{p_{t}: n}^{2}(\delta \eta, \delta \phi)$ in equation (1) for central $\mathrm{Au}-\mathrm{Au}$ collisions and four collision energies: $\sqrt{s_{\mathrm{NN}}}=19.6,62.4,130$ and $200 \mathrm{GeV}$. The increase of fluctuation amplitudes between 19.6 and $200 \mathrm{GeV}$ in figure 1 is a factor four, establishing that the $\left\langle p_{t}\right\rangle$ variance difference is strongly energy dependent. However, $\left\langle p_{t}\right\rangle$ fluctuations are difficult to interpret, whereas the corresponding $p_{t}$ angular autocorrelations obtained by inverting fluctuation scale dependence clearly indicate the underlying dynamics.

Figure 2 shows $p_{t}$ angular autocorrelations (by construction symmetric about $\eta_{\Delta}, \phi_{\Delta}=0$ ) of density ratio $\Delta \rho / \sqrt{\rho_{\text {ref }}}$ inferred from $\left\langle p_{t}\right\rangle$ fluctuation scale dependence as in figure 1 by inverting equation (2) [23]. The plots in figures 1 and 2 contain equivalent information in different forms, consistent with equation (2). One can observe the equivalents of the elliptic flow sinusoids in figure 2 (right panels) along the upper-right edges of the plots in figure 1 . Note that the elliptic flow sinusoid amplitudes in this autocorrelation representation using perparticle correlation measure $\Delta \rho / \sqrt{\rho_{\text {ref }}}$ are negligible for peripheral collisions and increase with increasing centrality towards a maximum for mid-central collisions. That behaviour contrasts with the nearly opposite trend observed with conventional per-pair measure $v_{2}$. The top two panels of figure 2 represent $130 \mathrm{GeV}$ and the bottom two panels $62.4 \mathrm{GeV} \mathrm{Au}-\mathrm{Au}$ collisions. The left panels represent comparable peripheral collisions and the right panels comparable central collisions. Autocorrelations for $200 \mathrm{GeV}$ are presented in [17], and the available $19.6 \mathrm{GeV}$ data do not have sufficient statistics for a satisfactory fluctuation inversion. The autocorrelations in figure 2 have same-side $\left(\left|\phi_{\Delta}\right|<\pi / 2\right)$ and away-side $\left(\left|\phi_{\Delta}\right|>\pi / 2\right)$ components.

We interpret the same-side peak and away-side ridge which dominate peripheral collisions as consistent with minijets as the source mechanism. The shapes are similar to jet correlations observed in p-p collisions [29], and in Au-Au collisions of all centralities as modelled by Hijing [24]. Those structures are strongly modified with increasing A-A centrality, but in a

\footnotetext{
${ }^{53}$ Quantity $v \approx 2 N_{\text {bin }} / N_{\text {part }}$, where $N_{\text {bin }}$ is the average number of $\mathrm{N}-\mathrm{N}$ collisions experienced by a participant nucleon and $N_{\text {part }}$ is the average participant nucleon number, estimates the mean participant path length in number of encountered nucleons.
} 

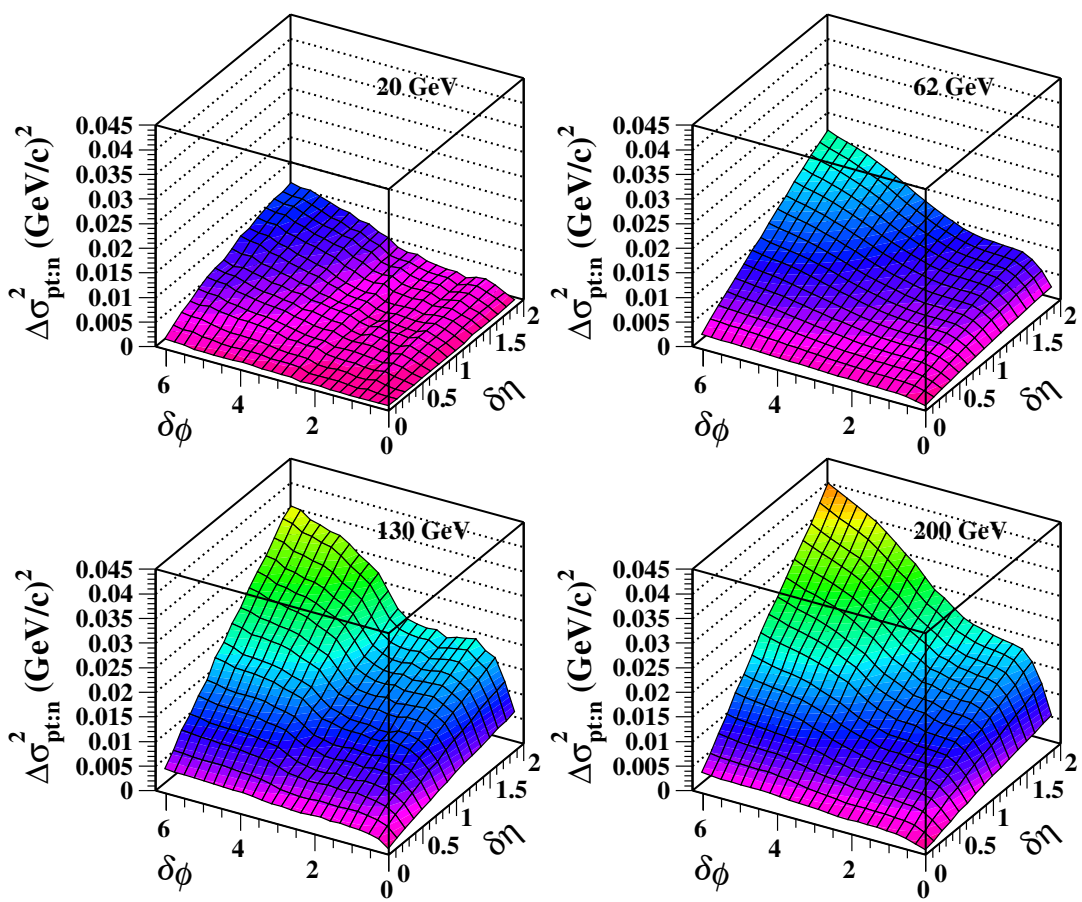

Figure 1. Per-particle $\left\langle p_{t}\right\rangle$ fluctuation scale dependence for 19.6, 62.4, 130 and $200 \mathrm{GeV} \mathrm{Au}-\mathrm{Au}$ collisions, and for the top $0-20,0-5,0-15$ and $0-5 \%$ of the total hadronic cross section respectively.

continuous manner which suggests that the minijet interpretation is also appropriate in central heavy ion collisions. By 'minijet' we refer to correlated hadrons from initial-state semi-hard parton scattering and subsequent fragmentation in which no leading or trigger particle is required, i.e., fragments from minimum-bias partons with no analysis restriction placed on the parton momentum spectrum. We expect minimum-bias partons to be dominated by the low- $Q^{2}$ 'minijets' described by theory [1-4]. The amplitudes of the peripheral same-side peaks are notably similar for 62.4 and $130 \mathrm{GeV}$, whereas for central collisions the same-side peak amplitude increases strongly with energy, and the peak is significantly broadened in the $\eta_{\Delta}$ direction.

\section{Experiment comparisons}

The autocorrelations in figure 2 represent all the angular correlation information obtainable from corresponding fluctuation measurements. Different experimental circumstances (e.g., detector acceptances) may result in apparently conflicting fluctuation measurements. However, per-particle fluctuation measurements such as those presented here are exactly comparable at the same bin size or scale (integration limits of equation (2)), independent of detector geometry and other experimental details, because they integrate the underlying autocorrelations which are detector-independent distributions. $\left\langle p_{t}\right\rangle$ fluctuations have been measured by several collaborations $[12,13,17,18,21,22]$. PHENIX measurements at 130 and $200 \mathrm{GeV}$ [13] are compatible with STAR measurements at equivalent acceptances (scales). We wish to determine the energy dependence of $p_{t}$ angular corrrelations over the largest energy interval possible, from $200 \mathrm{GeV}$ at RHIC down to the lowest SPS energies. 

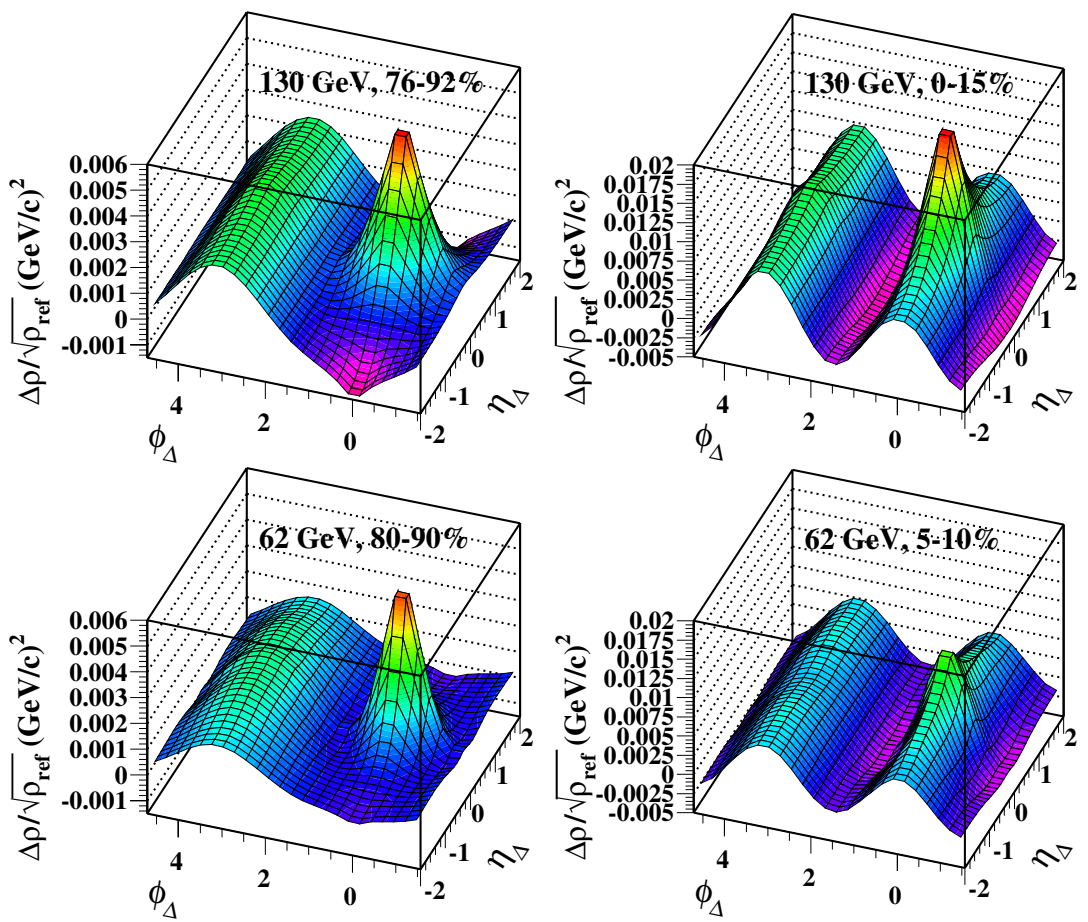

Figure 2. $p_{t}$ autocorrelations for $130 \mathrm{GeV}$ (upper) and $62.4 \mathrm{GeV}$ (lower), and for comparable peripheral (left) and central (right) $\mathrm{Au}-\mathrm{Au}$ collisions. These autocorrelations contain same-side $\left(\left|\phi_{\Delta}\right|<\pi / 2\right)$ and away-side $\left(\left|\phi_{\Delta}\right|>\pi / 2\right)$ structures.

However, the CERES SPS measurements are restricted to the $\eta$ scale dependence of $\left\langle p_{t}\right\rangle$ fluctuations at full azimuth. We make the most differential comparisons possible with that limited information, given that fluctuation scale dependence is the running integral of the underlying autocorrelation. First, we make a detailed comparison of STAR and CERES fluctuation scale dependence at full azimuth and equivalent pseudorapidity scales. Then we examine changes in centrality dependence with energy.

\subsection{Scale dependence}

Figure 3 (left panel) shows CERES $\Phi_{p_{t}}$ values for $\mathrm{Pb}-\mathrm{Au}$ collisions at several pseudorapidity scales and $2 \pi$ azimuth acceptance for $\sqrt{s_{\mathrm{NN}}}=12.3$ and $17.3 \mathrm{GeV}$ [18]. Also plotted are comparable STAR measurements of $\Delta \sigma_{p_{t}: n}$. The CERES data rise rapidly to about $3 \mathrm{MeV} / c$ within $\delta \eta \leqslant 0.2$ (see the inset), with a slower linear rise thereafter. STAR $19.6 \mathrm{GeV}$ data show similar behaviour, albeit with somewhat larger magnitudes over a larger $\eta$ acceptance. The higher-energy STAR data are qualitatively larger in magnitude. We attribute the rapid rise of CERES data $(\sim 0.003 \mathrm{GeV} / c)$ in $\delta \eta<0.2$ to quantum (HBT) and Coulomb correlations (resonance decays make a negligible contribution to $\left\langle p_{t}\right\rangle$ fluctuations) and designate those contributions as small-scale correlations (SSC). The complementary region of $\delta \eta$ then represents large-scale correlations (LSC). We conclude (see below) that the LSC component is dominated by parton fragments (including the away-side $\eta_{\Delta}$-independent azimuth peak), and possibly global temperature fluctuations (with corresponding autocorrelation uniform on $\left.\left(\eta_{\Delta}, \phi_{\Delta}\right)\right)$. 

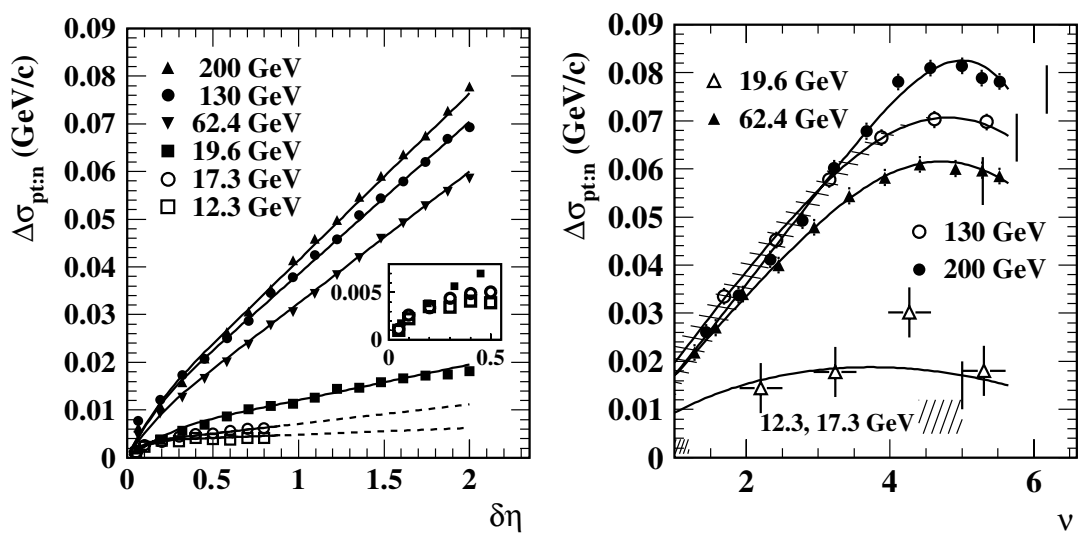

Figure 3. Left panel: per-particle fluctuation dependence on pseudorapidity scale $\delta \eta$ (in $2 \pi$ azimuth) in central collisions (cf figure 1 caption for STAR centralities). STAR measurements are solid symbols, CERES measurements [18] are open symbols. The inset shows details at small $\delta \eta$. Right panel: centrality dependence of $\left\langle p_{t}\right\rangle$ fluctuations in the STAR acceptance for four energies. $v$ is the mean participant path length (see footnote 53). The vertical line at right estimates $v$ for $b=0$. The upper hatched band estimates the uncertainty in $v$ for $130 \mathrm{GeV}$ data. There is an overall $14 \%$ systematic error in the corrected amplitudes. SPS measurements of $\Phi_{p_{t}}$ at 12.3 and $17.3 \mathrm{GeV}$ (the lower-right hatched region, with errors and centrality range) are included for comparison. Curves guide the eye.

\subsection{Centrality dependence}

Figure 3 (right panel) shows the centrality dependence of charge-independent $\left\langle p_{t}\right\rangle$ fluctuations (elliptic flow does not contribute to $\left\langle p_{t}\right\rangle$ fluctuations integrated over a $2 \pi$ azimuth acceptance). $\Delta \sigma_{p_{t}: n}$ was measured at the STAR acceptance scale and at four collision energies and corrected for tracking inefficiency and background contamination as in [12]. The vertical scale in this figure represents r.m.s. fluctuations measured by $\Delta \sigma_{p_{t}: n}$ (STAR). Corresponding $\Phi_{p_{t}}(\mathrm{CERES})$ values are numerically the same within $1.5 \%$ (less than the relative errors) within the range of the CERES data [18], and are therefore plotted without correction on the same scale.

The fluctuation amplitudes in figure 3 (right panel) vary strongly with collision centrality (consistent with [12]) and energy. The observed trends are fully consistent with $p_{t}$ angular autocorrelations reported in [17] (specifically the near-side minijet peak amplitude). Although there is a trend of monotonic increase with energy for the more central collisions, there is an interesting saturation of $\sqrt{s_{\mathrm{NN}}}$ dependence for peripheral collisions $(v \leqslant 2.5)$, consistent with the similarity between left panels in figure 2. Also included in this panel is a summary (lower-right hatched box) of $\Phi_{p_{t}}$ measurements for $\mathrm{Pb}-\mathrm{Au}$ collisions at 12.3 and $17.3 \mathrm{GeV}$ extrapolated to the STAR $\eta$ acceptance (see left panel) for comparison. The lower-left hatched box at $v=1$ represents a $\Phi_{p_{t}}$ measurement of $2.2 \pm 1.5$ (sys) $\mathrm{MeV} / \mathrm{c}$ for $\mathrm{p}-\mathrm{p}$ collisions at $17.3 \mathrm{GeV}$ (in the forward rapidity acceptance $y_{\pi} \in[1.1,2.6]$ ) [22]. We attribute no special significance to the apparent gap between 19.6 and $62.4 \mathrm{GeV}$ data in figure 3 . In figure 4 the data are consistent with the simple logarithmic trend $\ln \left(\sqrt{s_{\mathrm{NN}}}\right)$. The gaps in figure 3 arise from a conspiracy of currently available energies and the logarithmic trend.

\section{Errors}

Statistical errors for $\left\langle p_{t}\right\rangle$ fluctuation data in figure 1 are $0.005,0.001,0.003$ and $0.0015(\mathrm{GeV} / c)^{2}$ respectively for $19.6,62.4,130$ and $200 \mathrm{GeV}$ (statistical variations at 

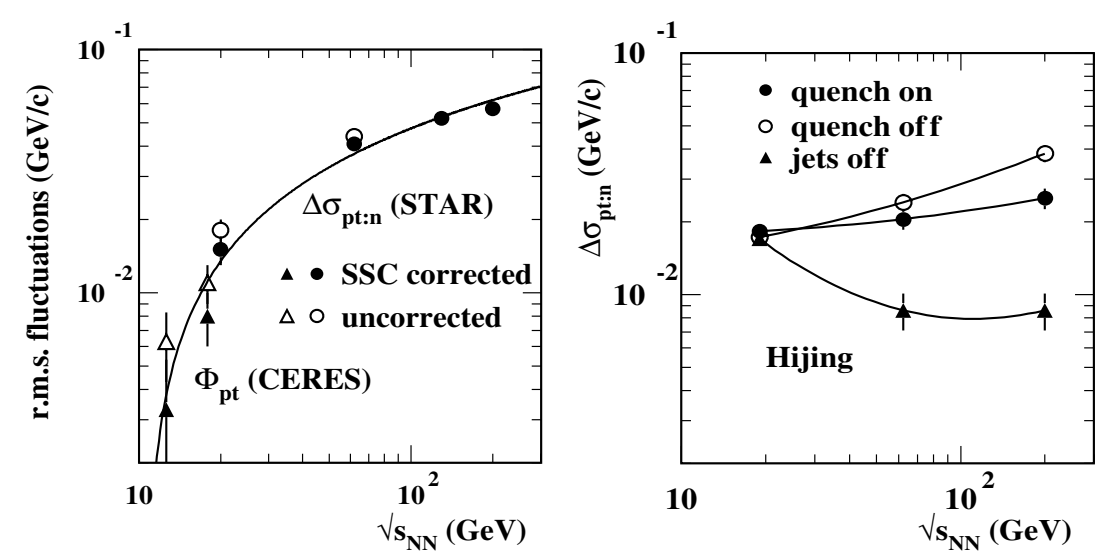

Figure 4. Left panel: $\sqrt{s_{\mathrm{NN}}}$ dependence of $\left\langle p_{t}\right\rangle$ fluctuations for central collisions and STAR full acceptance. CERES fluctuation data $(12.3$ and $17.3 \mathrm{GeV})$ were linearly extrapolated to the STAR $\eta$ acceptance (cf figure 3 ). The curve is proportional to $\ln \left\{\sqrt{s_{\mathrm{NN}}} / 10\right\}$. Right panel: Hijing-1.37 $\left\langle p_{t}\right\rangle$ fluctuations for central collisions and the STAR acceptance at three energies, for quench-on, quench-off and jets-off collisions.

different scale points are correlated by the integral nature of fluctuation scale dependence). Errors for fluctuation measurements in figure 3 (right panel) are indicated by error bars. The upper hatched band in figure 3 (right panel) estimates the uncertainty in $v$ for $130 \mathrm{GeV}$ data and provides an upper limit for 62.4 and $200 \mathrm{GeV} v$ uncertainties (more typically $<3 \%$ ). The uncertainty in $v$ for the $20 \mathrm{GeV}$ data is approximately 0.3 , as shown by horizontal errors in figure 3 (right panel). Systematic corrections to fluctuation amplitudes for tracking inefficiency and backgrounds vary over 15-22\% and 21-35\% ranges for 130 and $200 \mathrm{GeV}$ respectively. The overall systematic uncertainty for corrected fluctuation amplitudes is $14 \%$. Autocorrelation errors have two components: statistical fluctuations which survive smoothing and systematic error due to smoothing distortion. Statistical errors for the autocorrelations, estimated by inverting the error estimate for $\Delta \sigma_{p_{t}: n}^{2}$, are less than 0.0001 and $0.0003(\mathrm{GeV} / c)^{2}$ for autocorrelations at 62.4 and $130 \mathrm{GeV}$ respectively (bin errors are correlated). Smoothing distortions, estimated by passing data through inversion twice, are less than $5 \%$ of the range of autocorrelation values in each panel.

\section{Energy dependence}

As noted previously, although the energy dependence of $p_{t}$ angular autocorrelations has been established for STAR data by inversion of $\left\langle p_{t}\right\rangle$ fluctuations (e.g., figure 2 and [17]) $p_{t}$ autocorrelations have not been measured at SPS energies. Therefore, we infer the energy dependence indirectly using $\left\langle p_{t}\right\rangle$ fluctuation measurements as proxies. The energy dependence of $\left\langle p_{t}\right\rangle$ fluctuations for STAR and CERES data is summarized in figure 4. Fluctuations measured by the most-central, full-acceptance STAR $\Delta \sigma_{p_{t}: n}$, and CERES $\Phi_{p_{t}}$ values linearly extrapolated to $\delta \eta=2$ (CERES data are linear on $\delta \eta$ in [0.3,0.8], cf figure 3-left panel), are plotted in the left panel versus $\sqrt{s_{\mathrm{NN}}}$, with (solid points) and without (open points) SSC correction. As linear extrapolations the CERES points are actually upper limits, since an NA49 measurement at $17.3 \mathrm{GeV}$ in the pion rapidity interval $[1.1,2.6]$ in the $\mathrm{CM}$ gave an upper limit for $\Phi_{p_{t}}$ of $1.6 \mathrm{MeV} / c$ for central collisions [21]. A subsequent measurement in the same rapidity interval revealed nonzero results for more peripheral collisions [22]. The SSC correction consists of subtracting $0.003 \mathrm{GeV} / c$ from CERES $\Phi_{p_{t}}$ and STAR $\Delta \sigma_{p_{t}: n}$ 
values (the contribution to the integral of equation (2) for $\delta \eta<0.2$ ). Given the uncertainly in the SSC correction and the CERES extrapolations the open points at and below $19.6 \mathrm{GeV}$ represent upper limits on a minijet contribution. The solid curve, proportional to $\ln \left\{\sqrt{s_{\mathrm{NN}}} / 10\right\}$, summarizes the trend of the data above $10 \mathrm{GeV}$. We observe a dramatic increase of $\left\langle p_{t}\right\rangle$ fluctuations with beam energy for corrected and uncorrected data from an apparent onset of minijet-related correlation structure near $10 \mathrm{GeV}$.

The fluctuation measure $\Sigma_{p_{t}}=\sqrt{\Delta \sigma_{p_{t}: n}^{2} / \bar{n} \hat{p}_{t}^{2}}$ (quantities in the radicand are as defined in this paper) was employed in [18] to suggest that the energy dependence of $\left\langle p_{t}\right\rangle$ fluctuations is negligible, dominated by global temperature fluctuations with $\sigma_{T} / T_{0} \sim 1 \%$, unchanging from SPS to RHIC $[18,33] . \Sigma_{p}$ is based on four assumptions: (1) each collision event is thermalized with temperature $T,(2)$ an event ensemble has nonzero temperature variance $\sigma_{T}^{2}$ about ensemble mean $T_{0}$, (3) $\Delta \sigma_{p_{t}: n}^{2} / \bar{n}$ estimates $\sigma_{T}^{2}$ and 4) $\hat{p}_{t}$ estimates $T_{0} . \Sigma_{p_{t}}$ should therefore estimate $\sigma_{T} / T_{0}$. All four of those assumptions are falsified by the results reported in this paper. The event-wise thermalization scenario implied by assumptions (1) and (2) is falsified by the strong minijet $p_{t}$ correlations shown in figure 2 and in more detail in [17]. The observed $p_{t}$ correlations are almost three times larger than those predicted for no thermalization of jets (quench-off Hijing) in [24]. The separate elements in $\Sigma_{p_{t}}$ do not estimate temperature-related quantities as implied by assumptions (3) and (4). We learn from figure 2 and more extensive results in [17] that $\Delta \sigma_{p_{t}: n}^{2}$ is dominated by jet correlations when evaluated at $\delta \phi=2 \pi$ (the elliptic flow contribution then integrates to zero). The issue of parton scattering and incomplete equilibration is discussed further in section 8 .

Each of $\bar{n}, \Delta \sigma_{p_{t}: n}^{2}$ and $\hat{p}_{t}$ varies strongly with collision centrality and energy. Measured angular $p_{t}$ autocorrelations combined with two-component trends for $\bar{n}$ and $\hat{p}_{t}$ strongly suggest that those variations are dominated by incompletely-equilibrated semi-hard parton scattering. If the observed hard-scattering contribution were more strongly equilibrated $\Sigma_{p_{t}}$ might even fall sharply with increasing energy. The algebraic combination $\Sigma_{p_{t}}$ as measured happens to nearly cancel the $\sqrt{s_{\mathrm{NN}}}$-dependent hard-scattering trends of the individual factors, but the significance is unclear. A clearer picture emerges when the quantities are studied separately, as in the present study. $\Sigma_{p_{t}}$ is dominated by reference factor $1 / \sqrt{N_{\text {part }}}$ and mixes soft (HBT, Coulomb) and hard (parton fragment) contributions (SSC and LSC components respectively) through a running average on scale $\delta \eta . \Sigma_{p_{t}}$ is thus by construction insensitive to the observed energy dependence of parton scattering and fragmentation which dominates $\left\langle p_{t}\right\rangle$ fluctuations at RHIC and which motivated this study.

An analysis using a variant of $\Sigma_{p_{t}}$ denoted as $\sqrt{\left\langle\delta p_{t, i} \cdot \delta p_{t, j}\right\rangle} /\left\langle\left\langle p_{t}\right\rangle\right\rangle$ was reported in [34]. However, whereas CERES' $\Sigma_{p_{t}}$ is constructed with the same underlying statistical quantity $\Delta \sigma_{p_{t}: n}^{2}$ used in the present analysis, and is therefore directly comparable, the quantity $\sqrt{\left\langle\delta p_{t, i} \cdot \delta p_{t, j}\right\rangle} /\left\langle\left\langle p_{t}\right\rangle\right\rangle$ is not. In particular, $\left\langle\delta p_{t, i} \cdot \delta p_{t, j}\right\rangle$ includes in its denominator the random variable $n(n-1)$ (particle multiplicity $n$ varies randomly from event to event within some limits) [12]. For that reason it can produce results significantly inconsistent with $\Phi_{p_{t}}$ and $\Delta \sigma_{p_{t}: n}^{2}$ when bin multiplicities are small. For example, in figure 2 (lower-left panel) we show $p_{t}$ autocorrelations for $80-90 \%$ central $\mathrm{Au}-\mathrm{Au}$ collisions. The corresponding structure for the Hijing Monte Carlo is very similar: a same-side minijet peak and an away-side ridge from back-to-back jets [24]. However, the minijet peak from $\bar{n}\left\langle\delta p_{t, i} \cdot \delta p_{t, j}\right\rangle$ applied to the same Hijing data is $3 \times$ smaller, with additional (mainly negative) structure of comparable magnitude not seen in the $\Delta \sigma_{p_{t}: n}^{2}$ result.

Fluctuation measurements from application of $\bar{n}\left\langle\delta p_{t, i} \cdot \delta p_{t, j}\right\rangle$ and $\Delta \sigma_{p_{t}: n}^{2}$ to Hijing data for the full STAR detector acceptance and the range of centralities in [34] are consistent within the systematic errors reported in that paper, as expected from the correlations revealed by 
the present analysis. However, deviations in Hijing $p_{t}$ autocorrelations as described above become significant for centralities more peripheral than $50 \%$. Thus, $\left\langle\delta p_{t, i} \cdot \delta p_{t, j}\right\rangle$ cannot be used for a scaling analysis of $p_{t}$ fluctuations as described in this paper, where the average multiplicity in a bin can be as small as 1 .

Analysis of $\left\langle p_{t}\right\rangle$ fluctuations for Hijing-1.37 central collisions in the STAR acceptance at $200 \mathrm{GeV}[15,24]$ was extended down to 62.4 and $19.6 \mathrm{GeV}$ for this energy-dependence study. Values of $\Delta \sigma_{p_{t}: n}$ for quench-on, quench-off and jets-off central Hijing collisions (jet quenching modelled by pQCD gluon bremsstrahlung) are shown in figure 4 (right panel). The variation with energy of Hijing $\left\langle p_{t}\right\rangle$ fluctuations is small for jets-on (quench-off and quenchon) collisions. However, the corresponding Hijing $p_{t}$ autocorrelations (cf [24] for example at $200 \mathrm{GeV}$ ) reveal that the quench-off, near-side minijet peak amplitude falls by about $10 \times$ from 200 to $19.6 \mathrm{GeV}$, consistent with the variation of $\left\langle p_{t}\right\rangle$ fluctuations from data shown in the left panel. The source of the inconsistency is apparently the Hijing string fragmentation model. $p_{t}$ correlations in Hijing below $p_{t}=0.5 \mathrm{GeV} / c$ from string fragmentation persist even in central $\mathrm{Au}-\mathrm{Au}$ collisions and dominate $\left\langle p_{t}\right\rangle$ fluctuations in Hijing at lower energies, consistent with the jets-off results in the right panel. In contrast to Hijing we observe that string-related correlations in RHIC data are rapidly eliminated with increasing $\mathrm{Au}-\mathrm{Au}$ centrality, even for fairly peripheral collisions [35].

\section{Discussion}

Copious minijet production (a minijet 'plasma' [3]) has been predicted for Au-Au collisions at RHIC [1-5]. The theoretical concept of minijets is low- $Q^{2}$ partons (mainly gluons) produced in the initial stages of relativistic nuclear collisions [2]. The theoretical parton/minijet $Q$ range extends from a 'saturation' limit $Q_{s} \sim 1 \mathrm{GeV}$ for RHIC collisions up to several GeV (semi-hard) [3]. Minijets are said to carry most of the transverse energy in central A-A collisions at RHIC [4] and were believed (prior to these measurements) to equilibrate rapidly, driving experimentally-observable hydrodynamic phenomena (e.g., elliptic flow) [3]. Given the theoretical uncertainty and expected dominance of minijets in the early stages of RHIC collisions it is important to test the observability and degree of equilibration of low- $Q^{2}$ ( $Q \sim$ $1-5 \mathrm{GeV}$ ) partons.

Is a minijet interpretation allowed for these $p_{t}$ correlations? A perturbative model of minijet production in heavy ion collisions is stated to apply only above parton $p_{t} \sim 2 \mathrm{GeV} / c$ [19]. However, the authors also state that the $2 \mathrm{GeV} / c$ lower limit is only a limit on the theoretical description, not the physical phenomenon. And, fragments from $2 \mathrm{GeV} / c$ partons should appear at and below $1 \mathrm{GeV} / c$ hadron momentum. $\mathrm{p}-\overline{\mathrm{p}}$ fragmentation functions for parton energies up to $600 \mathrm{GeV}$ measured at FNAL extend down to $0.35 \mathrm{GeV} / c$ [36], and $\mathrm{e}^{+}-\mathrm{e}^{-}$fragmentation functions extend to much lower momenta $[37,38]$. The most probable fragment momentum in either case is $1-2 \mathrm{GeV} / c$ for a wide range of parton energies. Thus, there is no theoretical or observational reason which could preclude significant jet fragment contributions below $2 \mathrm{GeV} / c$ in heavy ion collisions. Conventional methods for measuring jet angular correlations based on a high- $p_{t}$ 'leading particle' are insensitive to partons below about $6 \mathrm{GeV}$. However, the novel analysis techniques developed for two-particle correlation analysis described in this paper and elsewhere [27-29, 35] have moved the threshold for direct observation of partons via final-state hadron correlations down to $Q \sim 1 \mathrm{GeV}$, and jet-like structure is indeed observed.

Is a minijet interpretation in fact necessary for these $p_{t}$ correlations? The correlation structure in figure 2 is dominated by a same-side peak, an away-side $\eta_{\Delta}$-independent ridge and a sinusoid. The sinusoid can be interpreted as elliptic flow (marking its first observation 
as a velocity phenomenon). A same-side peak (jet cone) and away-side ridge are the expected signature angular or number correlations for high- $p_{t}$ hadron fragments from hard-scattered partons. In [7] it was argued that high- $p_{t}$ angular correlations on azimuth $\phi$ obtained with the leading-particle method in A-A collisions are similar to those obtained in elementary $\mathrm{p}-\mathrm{p}$ and $\mathrm{e}^{+}-\mathrm{e}^{-}$collisions with full jet reconstruction and attributed to hard parton scattering. In this analysis the same structure is observed in $p_{t}$ correlations for $p_{t}<2 \mathrm{GeV} / c$ without a high- $p_{t}$ leading particle. Jet structure dominates peripheral collisions in figure 2 (left panels), and jet-like structure in central collisions (right panels), although strongly modified, is part of a continuous shape evolution from $\mathrm{N}-\mathrm{N}$ collisions.

Minijet structures have been observed as angular number correlations of low- $p_{t}$ particles in $\mathrm{Au}-\mathrm{Au}$ collisions at $130 \mathrm{GeV} \mathrm{[35]} \mathrm{and} \mathrm{p-p} \mathrm{collisions} \mathrm{at} 200 \mathrm{GeV}$ [29]. Low- $p_{t}$ jet-like structures in $p_{t}$ and number correlations are observed in Hijing Monte Carlo data where the correlation mechanism is known to be parton fragmentation [24]. In fact, the low- $p_{t}$ jet-like structure that we observe is exactly what is described as minijets by theory [39]. We conclude therefore that the analogous $p_{t}$ correlations in this analysis strongly support a minijet interpretation in which hadron fragments from minimum-bias partons (no condition is imposed by the analysis on the underlying parton momentum distribution) are peaked at low $p_{t}$. Ironically, low- $Q^{2}$ partons may be more precisely and unambiguously characterized by high-statistics $p_{t}$-autocorrelation studies than partons studied in conventional high- $p_{t}$ leadingparticle studies with their biased parton momentum spectra and background subtraction issues.

As noted, $\Delta \rho / \sqrt{\rho_{\text {ref }}}$ is within a constant $\left(\epsilon_{\eta} \epsilon_{\phi} / \sigma_{\hat{p}_{t}}^{2} \sim 0.25\right)$ Pearson's correlation coefficient, a measure of the relative covariance between fluctuations in pairs of bins. $\Delta \rho / \sqrt{\rho_{\text {ref }}}$ for number correlations measures the number of correlated particle pairs per detected particle. If $\left\langle p_{t}\right\rangle$ fluctuations are dominated by minijet correlations, as our measurements strongly suggest, then increase of $\Delta \rho / \sqrt{\rho_{\text {ref }}}$ means that either there are relatively more minijets (partons) per detected particle with the same $p_{t}$ structure and multiplicity, or the average multiplicity and/or total $p_{t}$ of minijets has increased, or both. That the minijet correlation structure in figure 2 is comparable in amplitude to that identified with elliptic flow suggests that the two dynamical processes have comparable importance in heavy ion collisions at RHIC energies. The trend with $\sqrt{s_{\mathrm{NN}}}$ in figure 4 (left panel) suggests strong increase of minijet-associated $p_{t}$ production at higher energies, and a limit on detectable parton production (correlated hadron fragments) at lower energies, with the apparent onset of observable parton fragments near $\sqrt{s_{\mathrm{NN}}}=10 \mathrm{GeV}$.

We now return to the question of parton scattering, minijets and equilibration in heavy ion collisions. The correlation structure of an evolving physical system can be used to track the equilibration process. Equilibrated or 'thermalized' systems exhibit a large range of correlation types and degrees, from a Bose condensate to an ideal gas. What is relevant for study of a particular system evolving from a non-equilibrium initial state toward equilibrium is changes in its correlation structure. In the present case parton fragment correlations observed in elementary $\mathrm{N}-\mathrm{N}$ collisions should be modified in heavy ion collisions depending on the extent and nature of the equilibration process. We can compare observed correlations in heavy ion collisions with the expectation for linear superposition of $\mathrm{N}-\mathrm{N}$ collisions in a Glauber representation of transparent nuclei as a limiting case. In this analysis we show that the abundant minijet structure associated with elementary $\mathrm{N}-\mathrm{N}$ collisions and exhibiting energy dependence consistent with QCD expectations for parton scattering survives to kinetic decoupling in central $\mathrm{Au}-\mathrm{Au}$ collisions. The structure is strongly modified, but the amplitude is still very significant compared to the $\mathrm{N}-\mathrm{N}$ reference. That result suggests that any claims of complete thermalization for RHIC heavy ion collisions should be reconsidered. 


\section{Summary}

In conclusion, we report measurements at several collision energies of $p_{t}$ angular autocorrelations on pseudorapidity and azimuth difference variables inferred by inverting the scale dependence of $\left\langle p_{t}\right\rangle$ fluctuations. We also report the energy dependence of the centrality and scale dependence of $\left\langle p_{t}\right\rangle$ fluctuations to provide comparisons with CERES measurements at SPS energies. The $p_{t}$ autocorrelation distributions are interpreted as consisting mainly of minimum-bias parton fragments (i.e., dominated by minijets). We examine energy dependence over the broadest possible interval by comparing STAR measurements to compatible CERES measurements at the CERN SPS. The results are consistent across energies and experiments: (1) excess $\left\langle p_{t}\right\rangle$ fluctuations increase fourfold from 20 to $200 \mathrm{GeV}$ for central $\mathrm{Au}-\mathrm{Au}$ collisions, but saturate above $62 \mathrm{GeV}$ for peripheral $\mathrm{Au}-\mathrm{Au}$ and $\mathrm{p}-\mathrm{p}$ collisions; (2) those fluctuations correspond mainly to $p_{t}$ angular correlations identified as minijets, which in central $\mathrm{Au}-$ Au collisions are strongly deformed relative to $\mathrm{p}-\mathrm{p}$ collisions (e.g., the same-side peak is broadened on $\eta_{\Delta}$ ); (3) $\left\langle p_{t}\right\rangle$ fluctuations thereby associated with initial-state parton scattering increase with energy proportional to $\ln \left\{\sqrt{s_{\mathrm{NN}}}\right\}$ above an onset of detectable parton fragments near $\sqrt{s_{\mathrm{NN}}}=10 \mathrm{GeV} . p_{t}$ autocorrelations thus reveal substantial parton fragment correlations surviving from initial-state scattering even in central $\mathrm{Au}-\mathrm{Au}$ collisions. Claims of complete thermalization in central HI collisions should be reexamined in light of these results. The strong energy dependence observed in these correlation and fluctuation data should motivate additional measurements at lower RHIC energies, spaced in energy according to the observed logarithmic trend, to investigate the onset of parton fragment production and the relationship of low- $Q^{2}$ parton scattering to the QCD phase boundary.

\section{Acknowledgments}

We thank the RHIC Operations Group and RCF at BNL, and the NERSC Center at LBNL for their support. This work was supported in part by the Offices of NP and HEP within the US DOE Office of Science; the US NSF; the BMBF of Germany; IN2P3, RA, RPL, and EMN of France; EPSRC of the United Kingdom; FAPESP of Brazil; the Russian Ministry of Science and Technology; the Ministry of Education and the NNSFC of China; IRP and GA of the Czech Republic, FOM of the Netherlands, DAE, DST, and CSIR of the Government of India; Swiss NSF; the Polish State Committee for Scientific Research; SRDA of Slovakia, and the Korea Science and Engineering Foundation.

\section{References}

[1] Kajantie K, Landshoff P V and Lindfors J 1987 Phys. Rev. Lett. 592527

[2] Mueller A H 2000 Nucl. Phys. B 572227

[3] Nayak G C, Dumitru A, McLerran L D and Greiner W 2001 Nucl. Phys. A 687457

[4] Eskola K J and Tuominen K 2001 Phys. Rev. D 63114006

[5] Gustafson Gosta 2001 Preprint hep-ph/0102109

[6] d'Enterria D 2003 Preprint nucl-ex/0309015

[7] Adler C et al (STAR Collaboration) 2003 Phys. Rev. Lett. 90082302

[8] Adler C et al (STAR Collaboration) 2002 Phys. Rev. Lett. 89202301

[9] Adams J et al (STAR Collaboration) 2003 Phys. Rev. Lett. 91072304

[10] Arsene I et al (BRAHMS Collaboration) 2003 Phys. Rev. Lett. 91072305

[11] Adler S S et al (PHENIX Collaboration) 2003 Phys. Rev. Lett. 91072303

[12] Adams J et al (STAR Collaboration) 2005 Phys. Rev. C 71064906

[13] Adcox K et al (PHENIX Collaboration) 2002 Phys. Rev. C 66024901 Tannenbaum M J (PHENIX collaboration) 2004 J. Phys. G: Nucl. Part. Phys. 30 S1367 
[14] Cf X-N Wang and Gyulassy Miklos 1992 Phys. Lett. B 282466 for discussion of transverse flow and minijets

[15] Liu Q J and Trainor T A 2003 Phys. Lett. B 567184

[16] Adams J et al (STAR Collaboration) 2004 Preprint nucl-ex/0408012

[17] Adams J et al (STAR Collaboration) 2006 J. Phys. G: Nucl. Part. Phys. 32 L37

[18] Adamová D et al (CERES Collaboration) 2003 Nucl. Phys. A 72797

[19] Gyulassy M and Wang X N 1994 Comp. Phys. Commun. 83307 Gyulassy M and Wang X N 1991 Phys. Rev. D 443501

[20] Gaździcki M and Mrówczyński St 1992 Z. Phys. C 54127

[21] Appelshäuser H et al (NA49 Collaboration) 1999 Phys. Lett. B 459679

[22] Anticic T et al (NA49 Collaboration) 2004 Phys. Rev. C 70034902

[23] Trainor T A, Porter R J and Prindle D J 2005 J. Phys. G: Nucl. Part. Phys. 31809

[24] Liu Q J, Prindle D J and Trainor T A 2006 Phys. Lett. B 632197

[25] Adams J et al (STAR Collaboration) 2006 Phys. Lett. B 634347

[26] Kenney J F and Keeping E S 1962 Mathematics of Statistics Pt 1, 3rd edn (Princeton, NJ: Van Nostrand)

[27] Prindle D J and Trainor T A 2005 Proc. MIT Workshop on Correlations and Fluctuations in Relativistic Nuclear Collisions (Cambridge, MA, 21-23 April 2005), J. Phys.: Conf. Ser. 27118

[28] Trainor T A and Prindle D J (STAR Collaboration) 2005 Proc. MIT Workshop on Correlations and Fluctuations in Relativistic Nuclear Collisions (Cambridge, MA, 21-23 April 2005), J. Phys.: Conf. Ser. 27134

[29] Jefferson Porter R and Trainor T A (STAR Collaboration) 2005 Proc. MIT Workshop on Correlations and Fluctuations in Relativistic Nuclear Collisions (Cambridge, MA, 21-23 April 2005), J. Phys.: Conf. Ser. 2798

[30] Ackermann K H et al 2003 Nucl. Instrum. Methods A 499624 (see other STAR papers in volume 499)

[31] Adler C et al 2001 Phys. Rev. Lett. 87112303 Adler C et al 2002 Phys. Rev. Lett. 89202301

[32] Trainor T A and Prindle D J 2004 Preprint hep-ph/0411217

[33] Sako R and Appelshäuser H (CERES/NA45) 2004 Proc. Quark Matter 2004, J. Phys. G: Nucl. Part. Phys. $30 \mathrm{~S} 1371$

[34] Adams J et al (STAR Collaboration) 2005 Phys. Rev. C 72044902

[35] Adams J et al (STAR Collaboration) 2004 Phys. Rev. C 73064907 (Preprint nucl-ex/0411003)

[36] Acosta D et al (CDF Collaboration) 2003 Phys. Rev. D 68012003

[37] Akrawy M Z et al (OPAL Collaboration) 1990 Phys. Lett. B 247 617-28

[38] Braunschweig W et al (TASSO Collaboration) 1990 Z. Phys. C 47187

[39] Wang X N 1992 Phys. Rev. D 461900 